Research Article

\title{
Generation of Cell-Like Images Using Euclidean Distance from Edge
}

\author{
Toru Hiraoka*, Kohei Maeda \\ Department of Information Systems, University of Nagasaki, 1-1-1, Manabino, Nagayo-chou, Nishisonogi-gun, Nagasaki-ken 851-2195, Japan
}

\author{
ARTICLE INFO \\ Article History \\ Received 06 November 2020 \\ Accepted 08 March 2021 \\ Keywords \\ Non-photorealistic rendering \\ cell \\ Euclidean distance \\ edge \\ clarification \\ automatic generation
}

\begin{abstract}
A non-photorealistic rendering method has been proposed to generate cell-like images in which cell-like patterns are represented in photographic images. Cell-like patterns are automatically generated by the change of density of photographic images. However, cell-like patterns are irregularly arranged. In this paper, we propose a method to arrange cell-like patterns along the edges of photographic images. In addition, our method can express cell membrane and cell nucleus in cell-like patterns more clearly than the conventional method. We improves the conventional method by using Euclidean distance from the edges. We show that appealing cell-like images can be generated by our method through experiments using various photographic images.
\end{abstract}

(C) 2021 The Authors. Published by Atlantis Press B.V. This is an open access article distributed under the CC BY-NC 4.0 license (http://creativecommons.org/licenses/by-nc/4.0/).

\section{INTRODUCTION}

Non-photorealistic rendering is a field of computer graphics that can generate effective illustrations and attractive artistic images. Some researchers have proposed non-photorealistic rendering methods to simulate art expression techniques and to develop unprecedented artistic approaches [1-7]. One of the unprecedented artistic approaches is a method to generating cell-like images by an iterative calculation using inverse iris filter from photographic images [8]. Cell-like patterns are composed of cell membrane and cell nucleus. Cell-like images are overlaid with cell-like patterns in photographic images. Cell patterns are automatically generated by the change of density of photographic images. However, cell-like patterns are irregularly arranged. Therefore, a method for aligning cell-like patterns in cell-like images has been proposed [9]. The conventional method [9] is implemented by synthesizing sine and cosine waves into photographic images. However, in the conventional method [9], cell-like patterns are mainly arranged in a grid pattern, and therefore the edge preservation of cell-like images is not high. Also, the actual cells are not arranged in the grid pattern.

In this paper, we propose a method to arrange cell-like patterns along the edges of photographic images. Our method also has the feature that the cell membrane and cell nucleus in cell-like patterns can be expressed more clearly than the conventional methods $[8,9]$. We improve the conventional method [8] by using Euclidean distance from the edges. Cell-like images of our method can give an impression different from cell-like images of the conventional methods [8,9]. By conducting experiments using various photographic images, it is visually confirmed that cell-like patterns can

“Corresponding author.Email: hiraoka@sun.ac.jp be generated along the edges and cell-like patterns that express the cell membrane and cell nucleus more clearly than the conventional methods [8,9] are generated. In addition, it is visually confirmed that the size of cell-like patterns can be changed by changing the values of the parameters in our method.

This paper is organized as follows: Section 2 describes our method to arrange cell-like patterns along the edges, Section 3 shows experimental results and reveals the effectiveness of our method, and the conclusion of this paper is given in Section 4.

\section{OUR METHOD}

Our method is implemented in two steps. In the first step, distance-transformed images are created by calculating Euclidean distance from the edges of photographic images. In the second step, cell-like images are generated using distance-transformed images and inverse iris filter. Inverse iris filter is calculated by the procedure that restores the transformed image using iris filter [10] to the original image using inverse filter [11]. The flow chart of our method is shown in Figure 1.

We explain details of the procedure in Figure 1.

Step 0: Let input pixel values of RGB on coordinates $(i, j)$ in a color photographic image be $f_{R, i, j}, f_{G, i, j}$ and $f_{B, i, j}(i=1,2,3, \ldots, I ; j=$ $1,2,3, \ldots, J)$. The pixel values $f_{R, i, j}, f_{G, i, j}$ and $f_{B, i, j}$ have value of $U$ gradation from 0 to $U-1$.

Step 1: Gray-scale pixel values $f_{i, j}$ are calculated as follows.

$$
f_{i, j}=\frac{f_{R, i, j}+f_{G, i, j}+f_{B, i, j}}{3}
$$




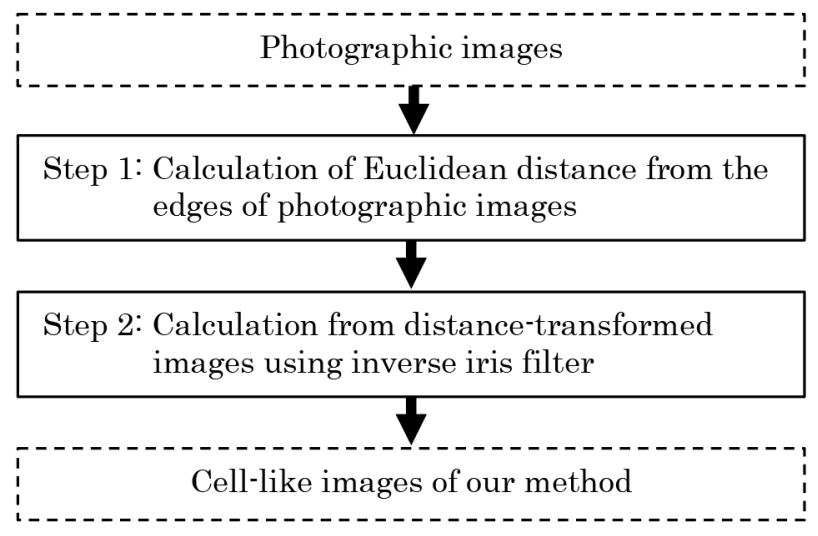

Figure 1 Flow chart of our method.

Edges are extracted from the gray-scale image using EDISON [12] that is a feature extraction tool that integrates edge detection and image segmentation. Shortest Euclidean distances $d_{1, i, j}$ from the edge pixels are calculated at each pixel.

If the Euclidean distances $d_{1, i, j}$ are within $m D+D / 2 \pm 0.5(m=0$, $1,2, \ldots)$, the Euclidean distances $d_{2, i, j}$ are set to 0 , where $D$ is a positive constant. Otherwise, the Euclidean distances $d_{2, i, j}$ are set to $\infty$. When the positions where the Euclidean distances $d_{2, i, j}$ are 0 are expressed on the image, it becomes lines of the interval $D$ along the edges.

Scan from the upper left to the lower right of the image, and in the case that the Euclidean distance $d_{2, i, j}$ are 0 at the target pixel $(i, j)$, the Euclidean distances $d_{2, k, l}$ in the range where the Euclidean distance from the target pixel $(i, j)$ is smaller than $D$ are updated to $\infty$. Where the pixels $(k, l)$ have the Euclidean distances from the target pixel $(i, j)$ smaller than $D$, and the Euclidean distance $d_{2, i, j}$ of the target pixel $(i, j)$ is not updated. When the positions where the Euclidean distances $d_{2, i, j}$ are 0 are expressed on the image, it becomes dotted lines with the spacing $D$.

Scan from the upper left to the lower right of the image, and in the case that the Euclidean distance $d_{2, i, j}$ are $\infty$ at the target pixel $(i, j)$, the Euclidean distance $d_{2, i, j}$ of the target pixel $(i, j)$ is updated to 0 if there is no pixel where the Euclidean distances $d_{2, k, l}$ are 0 in the range where the Euclidean distance from the target pixel $(i, j)$ is smaller than $D$. In all pixels $(i, j)$, there are pixels that the Euclidean distances $d_{2, k, l}$ are 0 within the radius $D$. Shortest Euclidean distances $d_{i, j}$ from the pixels that the Euclidean distances $d_{2, k, l}$ are 0 are calculated at each pixel. An image composed of the shortest Euclidean distances $d_{i, j}$ is called a distance-transformed image.

Step 2: Let output pixel values after processing with iris filter on $d_{i, j}$ be $\operatorname{IF}\left(d_{i, j}\right)$. Iris filter is executed with the $2 r+1$ peripheral pixels $(k, l)$ in the window of size $r$. Angles $\theta_{i, j, k, l}$ between a vector $(i-k, j-l)$ from the peripheral pixels $(k, l)$ to the target pixel $(i, j)$ and a vector $\left(\left(d_{k+2, l+2}+d_{k+2, l+1}+d_{k+2, l}+d_{k+2, l-1}\right.\right.$ $\left.+d_{k+2, l-2}\right)-\left(d_{k-2, l+2}+d_{k-2, l+1}+d_{k-2, l}+d_{k-2, l-1}+d_{k-2, l-2}\right),\left(d_{k+2, l+2}^{k+2, l-1}\right.$ $\left.+d_{k+1, l+2}+d_{k, l+2}+d_{k-1, l+2}+d_{k-2, l+2}\right)-\left(d_{k+2, l-2}+d_{k+1, l-2}+d_{k, l-2}+\right.$ $\left.\left.d_{k-1, l-2}+d_{k-2, l-2}\right)\right)$ are computed. Let convergence indices of the target pixel $(i, j)$ be $c_{i, j}$. The convergence indices $c_{i, j}$ are calculated as follows.

$$
c_{i, j}=\frac{1}{(2 r+1)^{2}}\left|\sum_{k=i-r}^{i+r} \sum_{l=j-r}^{j+r} \cos \theta_{i, j, k, l}\right|
$$

Let minimum and maximum values of $c_{i, j}$ in all pixels be $c_{\min }$ and $c_{\text {max }}$, respectively. The convergence indices $c_{i, j}$ are converted to $C_{i, j}$ as follows.

$$
C_{i, j}=255\left(\frac{c_{i, j}-c_{\text {min }}}{c_{\text {max }}-c_{\text {min }}}\right)
$$

The values $\operatorname{IF}\left(d_{i, j}\right)$ and $C_{i, j}$ are the same.

Pixel values $g_{R, i, j}, g_{G, i, j}$ and $g_{B, i, j}$ are computed by using inverse iris filter as follows.

$$
\begin{aligned}
& g_{R, i, j}=a\left(f_{, i, j}-C_{i, j}\right)+f_{R, i, j} \\
& g_{G, i, j}=a\left(f_{, i, j}-C_{i, j}\right)+f_{G, i, j} \\
& g_{B, i, j}=a\left(f_{, i, j}-C_{i, j}\right)+f_{B, i, j}
\end{aligned}
$$

where $a$ is positive constant. If $g_{R, i, j}, g_{G, i, j}$ and $g_{B, i, j}$ are less than 0 , then these values must be set to 0 , respectively. If $g_{R, i, j}, g_{G, i, j}$ and $g_{B, i, j}$ are greater than $U-1$, then these values must be set to $U-1$, respectively. The image composed of the pixel values $g_{R, i, j}, g_{G, i, j}$ and $g_{B, i, j}$ is a cell-like image of our method.

\section{EXPERIMENTS}

We conducted three experiments. In the first experiment, we visually confirmed the appearance of cell-like images generated from Lenna image shown in Figure 2 by changing the values of the parameters. In the second experiment, we applied our method to six photographic images in (Figure 3). All photographic images used in the experiments comprised $512 * 512$ pixels and 256 gradations. In the third experiment, we visually compared cell-like patterns generated by our method and the conventional methods [8,9]. For reference, the edge extracted image and the distance-transformed image $(D=15)$ of Lenna image are shown in Figure $4 \mathrm{a}$ and $4 \mathrm{~b}$, respectively.

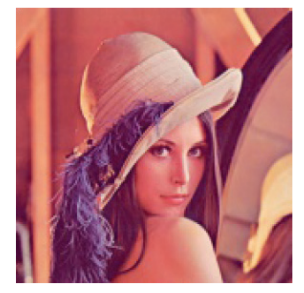

Figure 2 Lenna image.
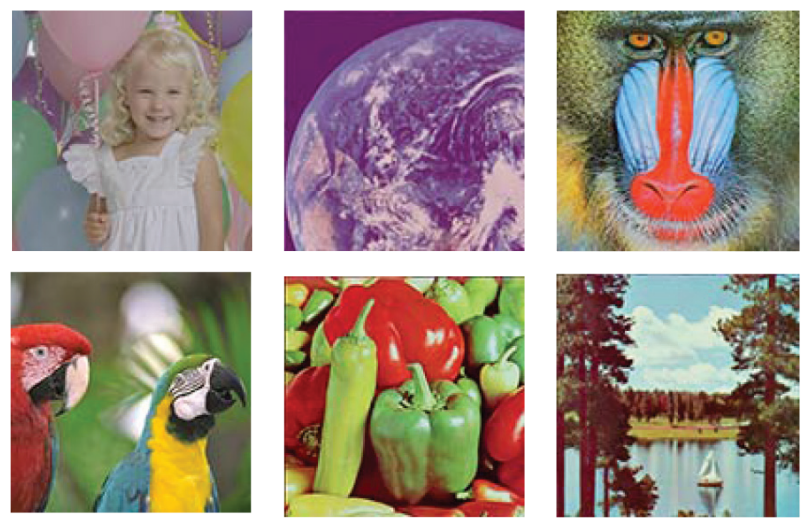

Figure 3 Various photographic images. 


\subsection{Experiments with Different Parameter Values}

We visually assessed the change in appearance of cell-like images as the value of the parameter $D$ was changed using Lenna image. The value of the parameter $D$ was set to 10,15 and 20 , and the value of the parameters $r$ and $a$ were set to 3 and 0.4 , respectively. Cell-like images in the case are shown in Figure 5. The larger the value of the parameter $D$, the larger cell-like patterns were expressed.

We visually assessed the change in appearance of cell-like images as the value of the parameter $r$ was changed using Lenna image. The value of the parameter $r$ was set to 2,3 and 4, and the value of the parameters $D$ and $a$ were set to 15 and 0.4 , respectively. Celllike images in the case are shown in Figure 6. The larger the value of the parameter $r$, the larger the cell nuclei of cell-like patterns were expressed.

We visually assessed the change in appearance of cell-like images as the value of the parameter $a$ was changed using Lenna image. The value of the parameter $a$ was set to 0.2, 0.4 and 0.6, and the value of the parameters $D$ and $r$ were set to 15 and 3, respectively. Cell-like images in the case are shown in Figure 7. The larger the value of the parameter $a$, the more clearly cell-like patterns were emphasized. a

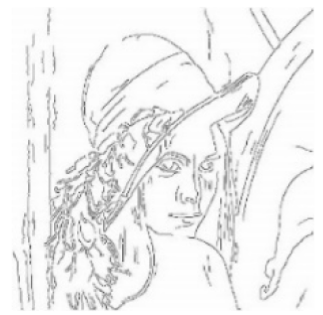

b

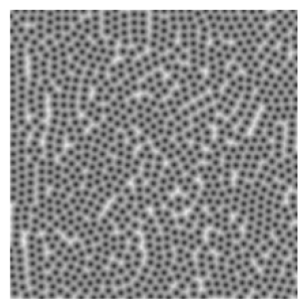

Figure 4 Edge-extracted image and distance-transformed image. (a) Edge-extracted image. (b) Distance-transformed image.
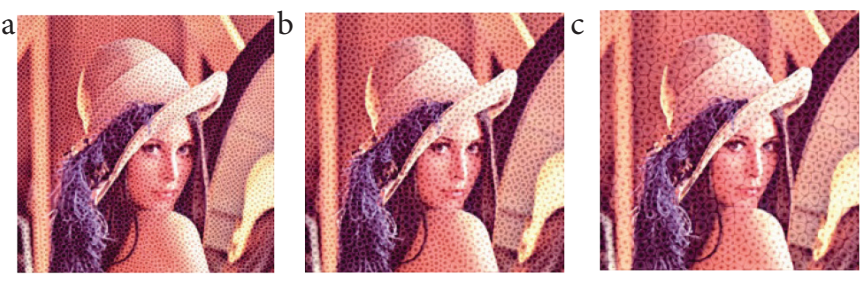

Figure 5 Cell-like images for $D=10,15$ and 20.
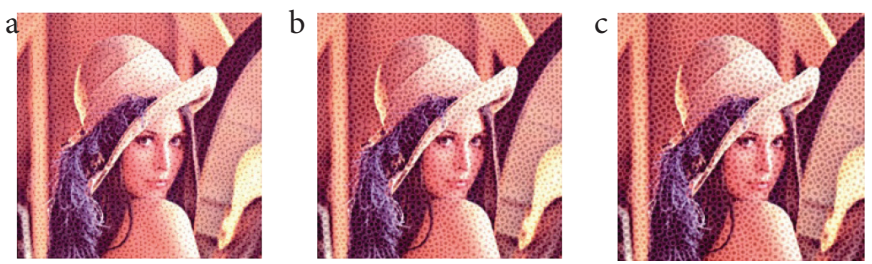

Figure 6 Cell-like images for $r=2,3$ and 4 .

\subsection{Experiments with Various Photographic Images}

We applied our method to six photographic images shown in Figure 3. Referring to the results of the experiments in the previous section, the values of the parameters $D, r$ and $a$ were set to 15,3 and 0.4 , respectively. Cell-like images generated from the six photographic images are shown in Figure 8. In all cell-like images, our method could express cell-like patterns along the edges of photographic images.

\subsection{Comparison Experiments with Conventional Methods}

We visually compared cell-like patterns generated by the conventional methods $[8,9]$. Cell-like images generated by the conventional methods [8,9] using Lenna image are shown in Figure 9. Our method could generate cell-like patterns more along the edges than the conventional methods $[8,9]$. In addition, the cell-like patterns generated by our method were expressed the cell membrane and cell nucleus more clearly than those of the conventional methods $[8,9]$.
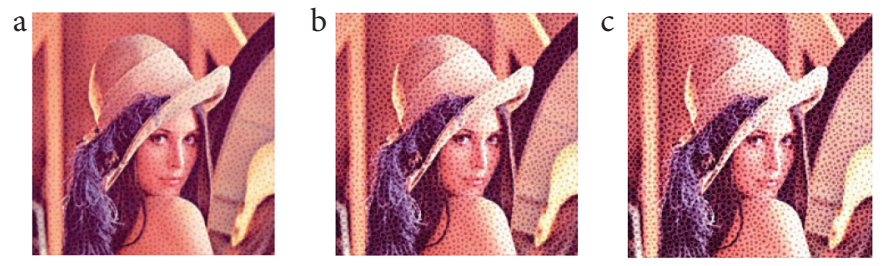

Figure 7 Cell-like images for $a=0.2,0.4$ and 0.6.
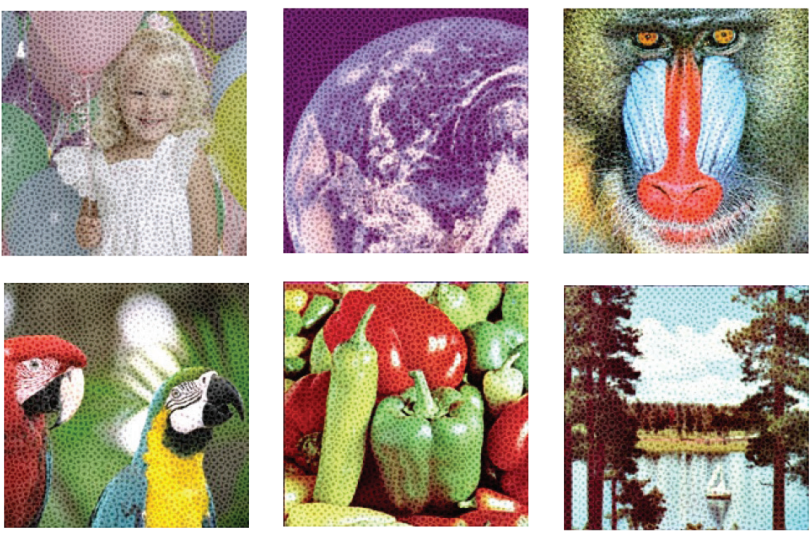

Figure 8 Cell-like images.
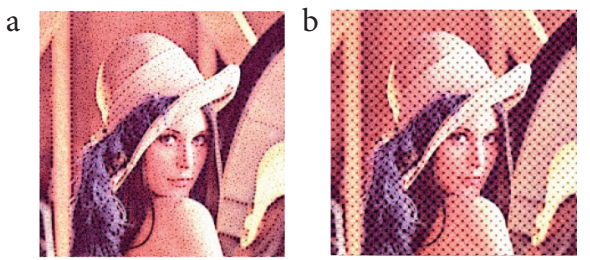

Figure 9 Cell-like images generated by the conventional methods [8,9]. 


\section{CONCLUSION}

We proposed a method to arrange cell-like patterns along the edges of photographic images. We improved the conventional method [8] by using Euclidean distance from the edges of photographic images. We demonstrated the effectiveness of our method through experiments using various photographic images. The experimental results showed that our method can express cell-like patterns along the edges and can express cell-like patterns with the cell membrane and cell nucleus in cell-like patterns more clearly than the conventional methods $[8,9]$. In addition, the experimental results showed that the size of cell-like patterns can be changed by changing the values of the parameters in our method.

In future work, we will try to apply our method to videos and three-dimensional data.

\section{CONFLICTS OF INTEREST}

The authors declare they have no conflicts of interest.

\section{ACKNOWLEDGMENT}

This work was supported by JSPS KAKENHI Grant Number JP19-K12664.

\section{REFERENCES}

[1] P. Haeberli, Paint by numbers: abstract image representations, ACM SIGGRAPH Comput. Graph. 24 (1990), 207-214.

\section{AUTHORS INTRODUCTION}

\section{Dr. Toru Hiraoka}

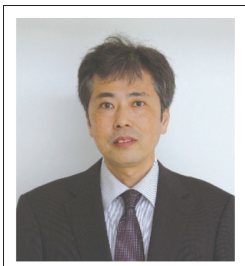

He graduated Doctor course at Design in Kyushu Institute of Design. He is a Professor in the Faculty of Information Systems in University of Nagasaki. His current research interests are non-photorealistic rendering and disaster prevention.
[2] D.D. Seligmann, S. Feiner, Automated generation of intent-based 3D illustrations, Proceedings of the 18th annual conference on Computer graphics and interactive techniques, 1991, pp. 123132.

[3] J. Lansdown, S. Schofield, Expressive rendering: a review of nonphotorealistic techniques, IEEE Comput. Graph. Appl. 15 (1995), 29-37.

[4] W. Qian, D. Xu, K. Yue, Z. Guan, Y. Pu, Y. Shi, Gourd pyrography art simulating based on non-photorealistic rendering, Multimed. Tools Appl. 76 (2017), 14559-14579.

[5] D. Martín, G. Arroyo, A. Rodríguez, T. Isenberg, A survey of digital stippling, Comput. Graph. 67 (2017), 24-44.

[6] W. Qian, D. Xu, J. Cao, Z. Guan, Y. Pu, Aesthetic art simulation for embroidery style, Multimed. Tools Appl. 78 (2019), 995-1016.

[7] T. Hiraoka, Generation of edge-enhancing labyrinth images using inverse filter and two improved Laplacian filters, ICIC Express Lett. 14 (2020), 121-127.

[8] T. Hiraoka, M. Hirota, K. Inoue, K. Urahama, Generating cell-like color images by inverse iris filter, ICIC Express Lett. 11 (2017), 399-404.

[9] T. Hiraoka, A method for emphasizing and aligning patterns in cell-like images, ICIC Express Lett. 13 (2019), 1031-1037.

[10] H. Kobatake, S. Hashimoto, Convergence index filter for vector fields, IEEE Trans. Image Process. 8 (1999), 1029-1038.

[11] Z. Yu, K. Urahama, Iterative method for inverse nonlinear image processing, IEICE Trans. Fundam. Electron. Commun. Comput. Sci. E97.A (2014), 719-721.

[12] The Robust Image Understanding Laboratory at Rutgers University, Edge detection and image segmentation (EDISON) system, 2002. Available from: http://sid.ethz.ch/debian/ orfeo-toolbox/OTB-3.20.0/Utilities/otbedison/help/doc/help. html.

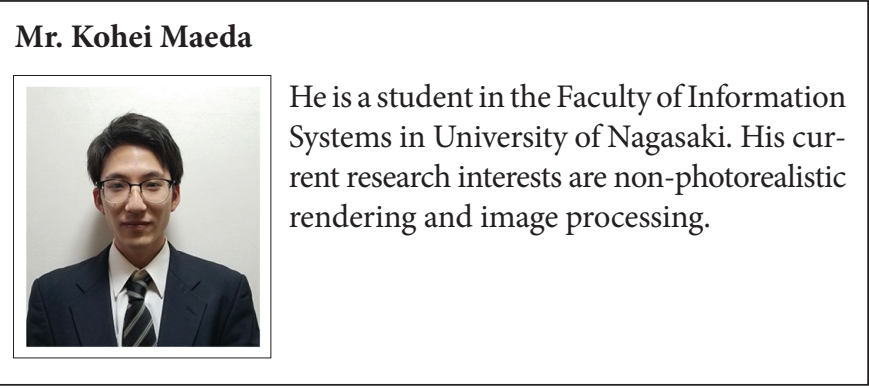

